\title{
ORIGINAL
}

\section{ACCESIBILIDAD A LOS SERVICIOS DE SALUD EN LA PRÁCTICA DE CITOLOGÍA RECIENTE DE CUELLO UTERINO EN UNA ZONA URBANA DE COLOMBIA (*)}

\section{Diego Iván Lucumí Cuesta y Luis Fernando Gómez Gutiérrez}

División de Salud Fundación FES-Social.

RESUMEN

Fundamento: El cáncer de cuello uterino es un problema de salud pública en países en desarrollo. Para cambiar esta situación se ha promovido la citología de cuello uterino. Sin embargo, su cobertura no es apropiada por múltiples factores. El objetivo de este artículo fue explorar la relación entre la práctica de citología de cuello uterino reciente -aquélla realizada tres años previos a la encuesta- el aseguramiento y el acceso a servicios de salud.

Métodos: Se analizaron 1.021 registros de mujeres de entre $18 \mathrm{y}$ 64 años, provenientes de un estudio transversal realizado en un área urbana de Bogotá (Colombia). Se indagó acerca de la fecha de última citología de cuello uterino, acceso a servicios de salud, afiliación al seguro de salud y variables sociodemográficas. Una vez recolectados los datos se realizó un análisis descriptivo y se construyó un modelo de regresión logística.

Resultados: El porcentaje de respuesta fue de $97,8 \%$. De 1.021 registros se excluyeron 38 , por ser mujeres con histerectomía. De las 983 restantes, $733(69,7 \%)$ reportaron una citología de cuello uterino reciente, que estuvo asociada a tener más de 30 años, residir en estrato socioeconómico medio, no ser soltera, tener alto nivel escolaridad, tener afiliación al seguro de salud y contar con una institución de salud a la que usualmente asiste cuando lo requiere.

Conclusiones: Estrategias de corto y mediano plazo dirigidas a incrementar el aseguramiento y acceso a servicios de salud son fundamentales para aumentar las coberturas de práctica citología de cuello uterino en países en desarrollo.

Palabras clave: Neoplasmas del cuello uterino. Prevención.

Correspondencia:

Diego Iván Lucumí Cuesta.

Carrera 7 No. 73-55 Oficina 1202.

Bogotá.

Colombia.

Teléfax: (57) 1-3130838, 3121130

Correo electrónico: dilucumi@ fundacionfes.org

(*) Los datos usados para este artículo fueron tomados del Estudio Santa Fe, el cual fue financiado por la Secretaria de Saluc de Bogotá D.C. (Colombia).

\section{ABSTRACT}

Accessibility to Healthcare Services in the Recent Cervical Cytology Performed in an Urban Area in Colombia

Background: Cervical cancer is a public health problem in developing countries. Cytology of the uterine cervix has been promoted to change this situation, the coverage thereof not being adequate for many different reasons. This study is aimed at delving into the relationship between the recent cervical cytology performed and that performed three years previous to the survey, insurance and access to healthcare services.

Methods: An analysis was made of 1,021 records of women within the 18-64 age range from a cross-sectional study conducted in an urban area of Bogota (Colombia). Research was conducted regarding the date of the last cervical cytology undergone, access to healthcare services, health insurance affiliation and socio-demographic variables. Once the data had been gathered, a descriptive analysis was conducted and a logic regression model was then constructed.

Results: A $97.8 \%$ response was achieved. A total of 38 of the 1,021 records were ruled out due to their being records of women having had hysterectomies. A total of $733(69.7 \%)$ of the other 983 records reported a recent cervical cytology, which was related to being over 30 years of age, being from a middle-class socioeconomic stratum, not being single, having a major degree of schooling, health insurance affiliation and having a health institution to which to go for care when needed.

Conclusions: Short-range and medium-range strategies aimed at increasing healthcare insurance and access to healthcare services are fundamental for increasing the coverage of performing cervical cytologies in developing countries.

Key words: Cervical cancer. Vaginal smear. Secondary preven- 


\section{INTRODUCCIÓN}

En el mundo se diagnostican cada año aproximadamente 466.000 nuevos casos de cáncer de cuello uterino (CCU), el cual representa el $10 \%$ de todos los cánceres diagnosticados en mujeres ${ }^{1}$. La enfermedad es considerada como un problema de salud pública, especialmente en países en desarrollo, donde se presentan el $80 \%$ de los casos diagnosticados y de las muertes a nivel mundial ${ }^{2,3}$. En estos países la enfermedad es la principal causa de muerte por cáncer en el sexo femenino, siendo el $90 \%$ de los casos en mujeres mayores de 35 años ${ }^{2,4}$.

De acuerdo a las estadísticas del Instituto Nacional de Cancerología, principal centro de referencia de cáncer en Colombia, de los 4.196 casos de cáncer diagnosticados en 1999 en mujeres y hombres de todas las edades, el 16,4\% correspondían a cuello uterino, ubicándolo como la principal localización de cáncer ${ }^{5}$. En este mismo año fue además la principal causa de muerte por neoplasias en la población femenina colombiana ${ }^{2}$.

Los estudios epidemiológicos realizados en los últimos 30 años han establecido una fuerte asociación entre el cáncer de cuello uterino y los comportamientos sexuales, entre los que se pueden identificar la edad temprana de inicio de relaciones sexuales y un elevado número de compañeros sexuales ${ }^{1}$. Otros factores asociados son la multiparidad, el consumo de tabaco y uso prolongado, por más de 12 años, de anticoncepción oral $^{1,2,6,7}$. Las condiciones socioeconómicas que predominan en las mujeres que presentan la enfermedad son: bajo nivel de escolaridad, bajo ingreso económico y baja cobertura de servicios de salud, en especial, los destinados a la prevención secundaria de este tipo de cáncer ${ }^{2,8,9}$.

La infección por el virus del papiloma humano (VPA) se ha identificado como un factor causal, en especial los serotipos 16 y 18 , que han sido calificados como carcinó- genos. Esta infección de transmisión sexual, que está asociada a algunos de los factores descritos, se encuentra presente en el 99,7\% de los casos de la enfermedad. Sin embargo, sólo el 5\% de las mujeres infectadas por este virus desarrollan la neoplasia ${ }^{1,2}$.

Desde los años 60, en una gran cantidad de países incluido Colombia, se ha promovido con fines de prevención secundaria, la realización de la citología cérvico uterina -CCU- o prueba de Papanicolau ${ }^{2,3,11}$. La recomendación sobre la periodicidad de realización de esta prueba depende de los recursos con los que se cuente para el desarrollo del programa de tamización y los hallazgos en la citología previa $^{2}$. Para los países con bajos recursos, se recomienda su práctica hasta cada 10 años, dando prioridad a las mujeres entre los 35 y 60 años, por ser las de mayor riesgo ${ }^{1}$. Este lapso entre una citología y otra permite una reducción potencial del $64 \%$ de las tasas acumuladas de cáncer cervicouterino, comparada con el 93\% que se obtiene cuando se realiza de forma anual o bianual y el $91 \%$ cuando se realiza cada trienio ${ }^{2}$.

En Colombia se aplica actualmente el esquema 1-1-3, el cual consiste en la realización de dos citologías anuales y luego cada tres años si los dos primeros reportes están dentro de límites normales ${ }^{12}$. Sin embargo, a pesar de los beneficios comprobados que tiene la realización de la CCU, en los países en desarrollo existen múltiples limitaciones para alcanzar coberturas adecuadas, de tal forma que se puedan obtener los beneficios que lograron los países desarrollados al aumentar las coberturas y frecuencias del cribado $^{1}$.

Los estudios realizados en países en desarrollo para prevenir la enfermedad, como es el caso de Colombia, se han centrado en la identificación de factores asociados a la infección por el $\mathrm{VPH}^{13,14}$, mientras otros se han centrado en establecer las limitaciones de los programas de cribado ${ }^{11}$. 
Sin embargo, recientemente se ha planteado la necesidad de identificar otros factores que influyan en el desarrollo de intervenciones poblacionales que busquen incrementar esta práctica preventiva, especialmente en mujeres que viven en condiciones de marginalidad $^{15}$.

El objetivo del presente trabajo es explorar la relación existente entre algunas variables de acceso a servicios de salud y variables sociodemográficas, con la realización reciente de la citología vaginal en mujeres de 18 a 69 años de una zona urbana de Bogotá.

\section{SUJETOS Y MÉTODO}

\section{Tipo de estudio, características de la población de referencia y diseño muestral}

Los datos analizados en este artículo fueron extraídos del Estudio Santa Fe, el cual consistió en una medición transversal con el objetivo de determinar la prevalencia de factores de riesgo de enfermedades crónicas no transmisibles, previa a la realización de intervenciones comunitarias de carácter multifactorial, con el enfoque definido en los proyectos CARMEN (Conjunto de Acciones para la Reducción Multifactorial de Enfermedades No Transmisibles), promovidos por la Organización Panamericana de la Salud $^{16,17}$.

El estudio fue llevado a cabo entre mayo y agosto de 2002 en dos localidades de Bogotá, (ciudad con 7 millones de habitantes ${ }^{18}$ y capital de la República de Colombia): Santa Fe como área demostrativa del Proyecto CARMEN y Tunjuelito como área control. La primera, ubicada en el centro de la ciudad, tiene una población de 110.000 habitantes, predominantemente de estratos socioeconómicos bajo y medio-bajo, de la cual aproximadamente el $30 \%$ corresponde a mujeres entre 18 y 64 años ${ }^{19}$. Tunjuelito esta situada en el sur de la ciudad y su población es de 190.000 habitantes, y comparte similitudes en sus características sociodemográficas con la localidad de Santa $\mathrm{Fe}^{20}$.

Previamente a la realización del estudio un Comité de Ética externo revisó y aprobó los contenidos del protocolo y los procedimientos de medición realizados.

La recogida de información se realizó por encuestadoras con experiencia puerta a puerta en estudios poblacionales, la encuesta indagaba sobre diversos factores de riesgo de comportamiento en salud. Se calculó una muestra poblacional, probabilística, de conglomerados y trietápica. El marco muestral del estudio estuvo formado por la población residente no institucionalizada de estratos bajos (estrato II) y bajos-medios (estrato III) de 15 a 69 años de la localidad de Santa Fe y de 15 a 29 años de la localidad de Tunjuelito. Para la definición del tamaño de la muestra se realizaron varias exploraciones, calculando las precisiones deseadas en estimaciones puntuales y en cambios generados en el tiempo, en función de las prevalencias de diferentes factores de riesgo. Al final de este proceso se calculó un tamaño de muestra de 3.050 mujeres, que incluía un ajuste por no respuesta del $12 \%$ y un efecto de diseño de 1,3 .

En el diseño fueron considerados como estrato de estudio cada uno de los niveles socioeconómicos mencionados anteriormente. La metodología de selección probabilística en cada estrato comprendió tres etapas básicas: a) unidades primarias de muestreo (UPM) conformadas por manzanas, b) selección de una subfracción de viviendas en las UPM de la muestra y, c) selección final de las unidades últimas de muestreo en las viviendas, en los grupos etarios del estudio (15-29, 30-49 y 50-69 años).

Para propósitos específicos de este artículo, los investigadores restringieron los análisis a la información obtenida de las 1.021 mujeres encuestadas entre 18 y 64 años de la localidad de Santa Fe. 
La variable dependiente de este estudio consistió en determinar si las mujeres encuestadas habían tenido recientemente una citología de cuello uterino. Para tal propósito se tuvieron en cuenta los criterios utilizados por el Segundo Estudio Nacional de Factores de Riesgo para Enfermedades Crónicas de Colombia -ENFREC II- ${ }^{11}$ y por Selvin y Brett ${ }^{15}$, los cuales definen la variable CCU reciente, como haber realizado dicha prueba de tamización durante los tres años previos, en contraste con haber tenido la prueba hace más de 3 años o nunca habérsela realizado.

De las mujeres que no cumplían con este criterio, 26 tenían una CCU hacía más de 3 años y 224 manifestaron no habérsela realizado nunca. Los investigadores decidieron considerarlas en un mismo grupo.

\section{Variables independientes}

Se incluyeron en el análisis como variables independientes las siguientes: edad, estrato socioeconómico, estado civil, nivel de escolaridad, principal actividad en los últimos 30 días, afiliación al Sistema General de Seguridad Social (SGSSS) y uso de una Institución Prestadora de Servicios de Salud (IPS).

Los grupos de edad fueron integrados en tres categorías: 18-29, 30-49 y 50-64 años. Los criterios de los puntos de corte de esta variable fueron definidos teniendo en cuenta el riesgo diferencial por edad en la aparición del cáncer de cuello uterino ${ }^{1,3}$. El estrato socioeconómico fue determinado por medio de la clasificación dada en las tarifas de servicios públicos de energía, agua, alcantarillado y teléfono, el cual se establece para cada una de las manzanas residenciales de la ciudad de Bogotá (categorías: I= muy bajo, II=bajo, III=medio-bajo, IV=medio, $\mathrm{V}=$ medio-alto $\mathrm{y}$ $\mathrm{VI}=$ alto). En la variable estado civil las categorías fueron: soltera, unión libre o casada, separada o divorciada y viuda.
El nivel de escolaridad fue agrupado en: primaria incompleta o ninguno (menos de 5 años aprobado), primaria completa (5 años aprobados), secundaria incompleta (más de 5 y menos de 11 años aprobados), secundaria completa (11 años aprobados) y otros niveles superiores (personas con algunos años de estudio técnicos o universitarios, independientemente si habían finalizado su aprendizaje o carrera). La principal actividad en los últimos 30 días fue integrada en las categorías: oficios del hogar y otras ocupaciones. Para esta última, se agruparon todas las restantes opciones que se presentan en la tabla 1 .

La afiliación al Sistema General de Seguridad Social en Salud fue determinada por medio de la siguiente pregunta: ¿Esta afiliada como cotizante o beneficiaria a una Entidad Promotora de Salud (EPS) o Administradora del Régimen Subsidiado (ARS)?. En la legislación colombiana, las EPS y ARS son entidades de aseguramiento en salud que asumen los siniestros en personas con capacidad de pago y en condiciones de pobreza, respectivamente. La variable uso de una Institución Prestadora de Servicios de Salud (IPS) específica cuando lo requiere, fue determinada por medio de la siguiente pregunta: ¿Existe un hospital, un centro de salud, clínica o consultorio, al que usted generalmente va cuando esta enferma o necesita asistencia?

\section{Análisis estadístico}

Se realizó un análisis descriptivo de las características sociodemográficas de las participantes. Las proporciones de la variable «citología de cuello uterino reciente» fueron descritas de acuerdo a las características seleccionadas. Se aplicó la prueba de chi $^{2}$ de independencia con el fin de identificar diferencias significativas en las distribuciones.

Con el propósito de identificar los factores asociados a tener una citología de cuello 
Tabla 1

Características sociodemográficas de las mujeres participantes en el estudio de 18 a 64 años de la localidad de Santa Fe. Bogotá DC 2002

\begin{tabular}{|c|c|c|}
\hline Variable & Frecuencia & $(\%) *$ \\
\hline \multicolumn{3}{|l|}{ Grupos de edad (años) } \\
\hline $18-29$ & 403 & 41,0 \\
\hline $30-49$ & 455 & 46,3 \\
\hline $50-64$ & 125 & 12,7 \\
\hline \multicolumn{3}{|c|}{ Estrato socioeconómico del segmento urbano } \\
\hline \multicolumn{3}{|c|}{ Bajo (II) } \\
\hline \multirow[t]{2}{*}{ Medio-bajo (III) } & 520 & 52,9 \\
\hline & 463 & 47,1 \\
\hline \multicolumn{3}{|l|}{ Estado civil } \\
\hline Soltero (a) & 300 & 30,5 \\
\hline En pareja(casado/unión libre) & 554 & 56,4 \\
\hline Separada/Divorciada & 87 & 8,8 \\
\hline Viuda & 42 & 4,3 \\
\hline \multicolumn{3}{|l|}{ Nivel de escolaridad } \\
\hline Primaria incompleta & 173 & 17,6 \\
\hline Primaria completa & 185 & 18,8 \\
\hline Secundaria completa o incompleta & 514 & 52,3 \\
\hline Otros estudios superiores & 111 & 11,3 \\
\hline \multicolumn{3}{|c|}{ Principal actividad en los últimos 30 días } \\
\hline \multicolumn{3}{|l|}{ Oficios del hogar } \\
\hline Trabajó & 407 & 41,4 \\
\hline Estudió y trabajó & 440 & 44,8 \\
\hline Buscó trabajo & 32 & 3,3 \\
\hline Estudió & 30 & 3,1 \\
\hline Pensionada, jubilada & 52 & 5,3 \\
\hline \multirow[t]{2}{*}{ Incapacitada } & 3 & 0,3 \\
\hline & 19 & 1,9 \\
\hline \multicolumn{3}{|l|}{ Afiliación al SGSSS ${ }^{\mathrm{a}}$} \\
\hline $\mathrm{Si}$ & 666 & 67,8 \\
\hline No & 315 & 32,0 \\
\hline No sabe/no responde & 2 & 0,2 \\
\hline \multicolumn{3}{|c|}{ Uso de una IPS ${ }^{b}$ especifica cuando lo } \\
\hline $\mathrm{Si}$ & 177 & 18,0 \\
\hline No & 4 & 0,4 \\
\hline No sabe/no responde & & \\
\hline
\end{tabular}

* Proporciones no ajustadas por pesos muestrales

a SGSSS=Sistema General de Seguridad Social en Salud.

b IPS= Institución Prestadora de Servicios de Salud

uterino, se llevó a cabo un análisis de regresión logística siguiendo los criterios de Hosmer y Lemeshow ${ }^{21}$. En una primera etapa se seleccionaron las variables cuyas razones de disparidad crudas presentaban una significación estadística menor de 0,25 para iniciar la construcción del modelo múltiple. Posterior- mente se examinó la colinearidad entre las variables seleccionadas, obteniendo el valor del factor incremental de la varianza (Vif) producido por cada variable cuando era introducida al modelo ${ }^{22,23}$. Se realizó un proceso stepwise forward, dejando en el modelo las variables cuyos coeficientes presentaban 
Tabla 2

Proporciones ajustadas de mujeres con citología de cuello uterino reciente, según características seleccionadas en la localidad de Santa Fe. Bogotá DC 2002

\begin{tabular}{|c|c|c|c|c|c|c|}
\hline \multirow{3}{*}{ Características } & \multicolumn{4}{|c|}{ Citología reciente } & \multirow{3}{*}{$\mathbf{E s}^{*}$} & \multirow{3}{*}{$\mathbf{p}^{\S}$} \\
\hline & \multicolumn{2}{|c|}{$\mathbf{S i}$} & \multicolumn{2}{|c|}{ No } & & \\
\hline & $\mathrm{n}^{*}$ & $\%$ & $\mathrm{n}^{*}$ & $\%$ & & \\
\hline Total de participantes & 733 & 69,7 & 250 & 30,3 & 1,5 & - \\
\hline \multicolumn{7}{|l|}{ Grupos de edad (años) } \\
\hline $18-29$ & 247 & 60,7 & 156 & 39,3 & 2,9 & \\
\hline $30-49$ & 383 & 83,4 & 72 & 16,6 & 1,7 & 0,00 \\
\hline $50-64$ & 103 & 85,0 & 22 & 15,0 & 4,1 & \\
\hline \multirow{2}{*}{\multicolumn{7}{|c|}{$\begin{array}{l}\text { Estrato socioeconómico del segmento urbano } \\
\text { Bajo (II) }\end{array}$}} \\
\hline & & & & & & \\
\hline \multirow[t]{2}{*}{ Medio-bajo (III) } & 371 & 73,4 & 149 & 26,6 & 1,9 & 0,02 \\
\hline & 362 & 80,2 & 101 & 19,8 & 2,1 & \\
\hline \multicolumn{7}{|l|}{ Estado marital } \\
\hline Soltero (a) & 163 & 54,9 & 137 & 45,1 & 3,6 & \\
\hline En pareja(casado/unión libre) & 460 & 83,1 & 94 & 16,9 & 1,8 & 0,00 \\
\hline Separada & 73 & 81,5 & 14 & 18,5 & 4,1 & \\
\hline Viuda & 37 & 89,5 & 5 & 10,5 & 4,8 & \\
\hline \multicolumn{7}{|l|}{ Nivel de escolaridad } \\
\hline Primaria incompleta & 128 & 72,7 & 45 & 27,3 & 3,9 & \\
\hline Primaria completa & 145 & 78,2 & 40 & 21,8 & 3,6 & 0,36 \\
\hline Secundaria completa o incompleta & 384 & 76,6 & 130 & 23,4 & 2,4 & \\
\hline Otros estudios superiores & 76 & 68,3 & 35 & 31,4 & 4,9 & \\
\hline \multicolumn{7}{|c|}{ Principal actividad en los últimos 30 días } \\
\hline \multicolumn{7}{|l|}{ Oficios del hogar } \\
\hline \multirow[t]{2}{*}{ Otras ocupaciones } & 316 & 79,9 & 91 & 20,1 & 2,3 & 0,01 \\
\hline & 417 & 71,6 & 159 & 28,4 & 2,1 & \\
\hline \multicolumn{7}{|l|}{ Afiliación al SGSSS ${ }^{\text {a }}$} \\
\hline $\mathrm{Si}$ & 516 & 78,3 & 150 & 21,7 & 1,9 & 0,03 \\
\hline No & 217 & 69,8 & 98 & 30,2 & 3,4 & \\
\hline \multicolumn{7}{|c|}{ Uso de una IPS ${ }^{b}$ especifica cuando lo requiere } \\
\hline \multirow{2}{*}{$\begin{array}{l}\mathrm{Si} \\
\mathrm{No}\end{array}$} & 617 & 77,6 & 185 & 22,4 & 1,7 & 0,00 \\
\hline & 113 & 65,6 & 64 & 36,4 & 3,9 & \\
\hline
\end{tabular}

* Número de observaciones absolutas.

‡ Error estándar absoluto. Debido a que la variable dependiente: citología vaginal reciente es dicotómica, el Es es igual tanto para las categorías Sí y

\$ Significación estadística con un intervalo de confianza del $95 \%$

El número de observaciones analizadas no es igual al número total de datos analizados (983) debido a la no respuesta (2 en afiliación al SGSSS y 4

en uso de una IPS específica cuando lo requiere)

a SGSSS=Sistema General de Seguridad Social en Salud.

una probabilidad de entrada $(\mathrm{Pe})$ menor a 0,05 y una probabilidad de retiro $(\mathrm{Pr})$ menor a 0,15 .

Posteriormente se evaluaron posibles interacciones entre las variables finales, sin encontrarse ninguna. Se realizaron ajustes adicionales por el efecto de conglomerados de las UPM, la estratificación de la muestra y el peso relativo por desproporción de las probabilidades de selección en las diferentes particiones.

Los análisis estadísticos fueron procesados en el programa Stata Intercool versión $8^{24}$.

\section{RESULTADOS}

El porcentaje de respuesta obtenido en la partición muestral de análisis fue del 97,8\%, lo cual equivale a 1.021 mujeres entre 18 a 64 años de edad, de las cuales 38 manifestaron tener antecedentes positivos de histerectomía, razón por la cual los análisis estadísticos se limitaron a las 983 mujeres restantes.

El promedio de edad en este grupo fue de $34,3$ años ( $\mathrm{DS}=11,9)$. En la tabla 1 se presentan las características seleccionadas de las participantes. En la tabla 2 se puede apreciar que el $69,7 \%$ de las mujeres reportó haberse 
Tabla 3

Razones de disparidad de tener una citología vaginal reciente en mujeres de 18 a 64 años de la localidad de Santa Fe de acuerdo a características seleccionadas. Bogotá DC 2002

\begin{tabular}{|c|c|c|c|c|c|c|}
\hline Variable & $\begin{array}{c}\text { Razones de } \\
\text { disparidad }^{\text {crudas }^{\dagger}}\end{array}$ & $\mathbf{p}$ & IC $95 \%$ & $\begin{array}{l}\text { Razones de } \\
\text { disparidad } \\
\text { ajustadas }^{\dagger *}\end{array}$ & $\mathbf{p}$ & IC $95 \%$ \\
\hline \multicolumn{7}{|l|}{ Edad (años) } \\
\hline $18-29$ & 1 & & & 1 & & \\
\hline $30-49$ & 3,25 & 0,00 & $2,27-4,65$ & 2,82 & 0,00 & $1,95-4,07$ \\
\hline $50-64$ & 3,66 & 0,00 & $1,83-7,30$ & 3,22 & 0,00 & $1,54-6,74$ \\
\hline \multicolumn{7}{|l|}{$\begin{array}{l}\text { Estrato socioeconómico del } \\
\text { segmento urbano }\end{array}$} \\
\hline Bajo (II) & 1 & & & 1 & & \\
\hline Medio-bajo (III) & 1,47 & 0,02 & $1,05-2,05$ & 1,82 & 0,00 & $1,27-2,61$ \\
\hline \multicolumn{7}{|l|}{ Estado marital } \\
\hline Soltero (a) & 1 & & & 1 & & \\
\hline \multicolumn{6}{|l|}{ Separada } & $2,22-5,22$ \\
\hline Viuda & $\begin{array}{l}3,62 \\
6,98\end{array}$ & $\begin{array}{l}0,00 \\
0,00\end{array}$ & $\begin{array}{c}1,93-6,76 \\
2,48-19,67\end{array}$ & $\begin{array}{l}3,23 \\
6,43\end{array}$ & $\begin{array}{l}0,00 \\
0,00\end{array}$ & $\begin{array}{c}1,65-6,31 \\
1,86-22,14\end{array}$ \\
\hline \multicolumn{7}{|l|}{ Nivel de escolaridad } \\
\hline Primaria incompleta & 1 & & & 1 & & \\
\hline Primaria completa & 1,34 & 0,30 & $0,75-2,38$ & 1,65 & 0,09 & $0,91-2,98$ \\
\hline Secundaria completa incomplet a & 1,23 & 0,42 & $0,73-2,06$ & 2,46 & 0,00 & $1,37-4,41$ \\
\hline & 0,80 & 0,50 & $0,42-1,52$ & 2,76 & 0,00 & $1,30-5,81$ \\
\hline \multicolumn{7}{|l|}{$\begin{array}{l}\text { Principal actividad en los } \\
\text { últimos } 30 \text { días }\end{array}$} \\
\hline Oficios del hogar & 1 & & & 1 & & \\
\hline Otras ocupaciones & 0,63 & 0,01 & $0,43-0,91$ & 0,74 & 0,13 & $0,50-1,09$ \\
\hline \multicolumn{7}{|l|}{ Afiliación al SGSSS $^{\mathrm{a}}$} \\
\hline No & 1 & & & 1 & & \\
\hline $\mathrm{Si}$ & 1,56 & 0,03 & $1,02-2,37$ & 1,63 & 0,03 & $1,04-2,54$ \\
\hline \multicolumn{7}{|l|}{$\begin{array}{l}\text { Uso de una IPS }{ }^{b} \text { especifica } \\
\text { cuando lo requiere }\end{array}$} \\
\hline No & 1 & & & 1 & & \\
\hline $\mathrm{Si}$ & 1,98 & 0,00 & $1,34-2,93$ & 1,99 & 0,00 & $1,27-3,11$ \\
\hline
\end{tabular}

† Número de observaciones 979

**Las razones de dispariada fueron ajustados por grupos de edad, estrato socioeconómico, estado civil, ocupación y el uso de una IPS especifica cuando lo requiere.

SGSSS=Sistema General de Seguridad Social en Salud

b IPS= Institución Prestadora de Servicios de Salud

sometido a la práctica de una CCU recientemente, siendo las proporciones mayores de este indicador en las mujeres con las siguientes características: edad entre 50 a 64 años $(85,0 \%)$, estrato socioeconómico mediobajo $(80,2 \%)$, estado marital viuda $(89,5 \%)$, nivel de escolaridad de primaria completa $(78,2 \%)$, oficios del hogar como principal actividad en los últimos 30 días $(79,9 \%)$, estar afiliada al SGSSS (78,3\%) y usar una IPS específica cuando lo requiere $(77,6 \%)$. Se observaron diferencias estadísticamente significativas en todas las variables, exceptuando el nivel de escolaridad.
Las razones de disparidad crudas de tener una citología vaginal reciente evidencian que las mujeres de 30 a 49 años y de 50 a 64 años tuvieron una posibilidad mayor con respecto a las de 18 a 29 años, al igual que las pertenecientes al estrato III con respecto al II, las que viven en pareja, separadas y viudas frente a las solteras, las afiliadas al SGSSS con respecto a las que no lo eran y las que usaban una IPS específica cuando lo requerían (tabla 3 ). Las mujeres que realizaban actividades diferentes a las del hogar tuvieron menos posibilidades de tener una citología reciente con respecto a las dedicadas a dicha actividad. 
Una vez ajustado el modelo por posibles variables confundidoras, se observa el mismo sentido de las asociaciones encontradas en el modelo crudo en las variables: grupos de edad, estrato socioeconómico, estado marital, afiliación al SGSSS, uso de una IPS específica cuando lo requiere. Se destaca el hecho de que se evidencian asociaciones significativas en los niveles de escolaridad no encontradas en el modelo crudo, y que además las razones de disparidad se incrementan progresivamente a medida que el nivel de escolaridad es mayor (tabla 3 ).

\section{DISCUSIÓN}

Este estudio evidencia que el $69,7 \%$ de las mujeres de 18 a 64 años de la localidad de Santa Fe reportaron haberse realizado una citología de cuello uterino recientemente. Cifra similar a la encontrada en 1998 en el ENFREC II $(68,4 \%)$, en el que usando la misma definición para la variable dependiente se indagó sobre esta práctica en mujeres 25 a 64 años residentes en zonas urbanas y rurales de Colombia ${ }^{11}$.

Aunque los estudios no son comparables, los hallazgos pueden estar evidenciando las limitaciones que existen en el país para lograr incrementos en las coberturas de CCU rápidos y significativos, las cuales pueden estar asociadas al alto porcentaje de oportunidades perdidas para su realización $(43,6 \%)^{11}$ y a limitaciones en el acceso a los servicios de salud, específicamente el de toma de CCU.

Los autores identifican dos limitaciones básicas en el presente estudio. La primera está referida a que la inferencia estadística sólo puede hacerse para la localidad de Santa Fe, sin embargo, los resultados pueden contribuir a orientar en otros centros urbanos de Colombia el diseño e implementación de programas tendentes a incrementar las coberturas de la CCU, teniendo en cuenta que comparten características socioeconómicas y culturales con esta loca$\operatorname{lidad}^{25,26}$.

La segunda limitación tiene que ver con el hecho de que la información relacionada con la práctica de citología fue autoreportada por las participantes. En este sentido y con respecto a prácticas preventivas, Newell y colegas concluyeron que los individuos que participan en estudios poblacionales como el aquí reportado, tienden a sobrestimar la adopción de este tipo de comportamientos, entre otras razones con el fin de dar una respuesta socialmente deseable, por sesgos de memoria, o por la falta de validez en las preguntas empleadas ${ }^{27}$.

Con respecto al primer sesgo se puede considerar que la prevalencia encontrada puede ser menor que la real, dada la dificultad para controlar este fenómeno. Frente al sesgo de memoria, los periodos usados para indagar sobre la fecha de realización de la última citología de cuello uterino contribuyeron a minimizar su efecto. Finalmente, con el fin de reducir las implicaciones derivadas de la falta de validez se emplearon preguntas utilizadas previamente en otros cuestionarios $^{11,28}$.

Una vez ajustado por afiliación a la seguridad social y por las variables sociodemográficas estudiadas, las mujeres que refirieron haber tenido la posibilidad de usar, cuando lo requirieron, una entidad que presta servicios de salud, tuvieron dos veces mayor posibilidad de práctica reciente de la CCU, hallazgo similar a lo encontrado en un estudio realizado por Selvin y Brett ${ }^{15}$. Este hallazgo es importante en países como Colombia, donde la afiliación al sistema de salud no es aún universal, teniendo en cuenta el alto costo que implica alcanzar esta meta muchas mujeres podrían continuar siendo marginadas de la práctica de dicha prueba.

A corto plazo, las acciones para incrementar la cobertura de la CCU pueden centrarse en eliminar limitaciones en el acceso a este 
tipo de servicios preventivos, fortaleciendo las actividades a nivel primario. Sin embargo, altas coberturas en la realización de la prueba son importantes sólo si el programa de prevención cumple el ciclo que comprende desde la captación de la mujer hasta la atención adecuada y oportuna cuando se diagnostica la neoplasia.

Siendo la condición de afiliada al sistema de seguridad social en salud un factor que contribuyó a explicar en forma positiva y significativa la práctica reciente de la CCU, las estrategias a medio plazo deberán ir enfocadas a incrementar la cobertura de afiliación a dicho sistema, especialmente en mujeres de bajo nivel socioeconómico y baja escolaridad, las cuales tienen, al igual que en otros estudios, menor prevalencia de práctica de esta prueba preventiva $a^{4,29,30}$.

La condición de marginalidad de muchas mujeres en países en desarrollo, que en este estudio puede verse reflejada en el bajo nivel socioeconómico y baja escolaridad, debe centrar de forma urgente la atención de los que toman las decisiones, dada su negativa y significativa correlación con la CCU, la cual puede prevenir entre un 20 a $60 \%$ de las muertes causadas por cáncer de cuello uterino en América Latina ${ }^{31}$. De otro lado, los hallazgos aquí presentados ayudan a generar evidencia adicional en cuanto a la gran carga de inequidad que produce la marginalidad, pues la población pobre no sólo presenta mayores factores de riesgo para enfermedades crónicas $^{32}$ sino que, adicionalmente, tiene una menor posibilidad para participar en los programas preventivos ${ }^{33}$.

Tres retos principales deberán ser abordados por el programa de prevención de este tipo de cáncer en Bogotá: incrementar las coberturas de práctica reciente de CCU empleando el esquema 1-1-3; garantizar la integralidad del programa de prevención y promover acciones que actúen sobre los factores de riesgo y condicionantes para la enfermedad.
Para la estructuración de servicios de salud adecuados a las características socioculturales y de afiliación a la seguridad social de la población de bajo nivel socioeconómico, son útiles los hallazgos de un estudio cualitativo realizado para establecer factores asociados a la práctica de CCU en mujeres de bajos recursos socioeconómicos en Bogotá, en el cual se identificó la necesidad de disponer de servicios que trabajen con las mujeres, tanto como con sus parejas, para aumentar el grado de normatividad social frente a la práctica del examen, incrementen los niveles de sensibilización y compromiso frente al comportamiento, y flexibilicen y humanicen la práctica de la prueba de tamización ${ }^{34}$.

Dado que la responsabilidad para garantizar la práctica de esta prueba a mujeres de bajos recursos fue asignada desde 1994 a entidades públicas y privadas ${ }^{35}$, estudios posteriores deberán evaluar los cambios que recientemente se han incorporado en el sistema de salud, consistentes en trasladar la responsabilidad y los recursos para la realización de la CCU exclusivamente a las entidades del sector público ${ }^{36}$. Dichos cambios pueden generar economías de escalas que fortalezcan a nivel local los programas de prevención de esta localización del cáncer, permitiendo un incremento en las coberturas de CCU.

En conclusión, a pesar de los esfuerzos realizados en el país para incrementar las coberturas de práctica de la CCU, éstas siguen sin ser óptimas, lo cual se explica en parte por las limitaciones del acceso a los servicios de salud. Sin embargo, teniendo en cuenta la alta frecuencia de este cáncer en la población colombiana, deben llevarse a cabo esfuerzos adicionales con el fin de incrementar estas coberturas, para poder lograr, como en los países desarrollados, disminuciones en la incidencia y la mortalidad por esta causa ${ }^{4,37}$.

Ampliar la afiliación al sistema de seguridad social en salud, como medida a medio 
plazo, y prestar en forma integral los servicios de prevención de esta neoplasia a corto plazo, generando estrategias que mejoren el nivel de confianza de las mujeres en las instituciones prestadoras de servicios de salud, son las dos estrategias identificadas a partir de los hallazgos aquí reportados para abordar el problema.

\section{BIBLIOGRAFÍA}

1. Sankaranarayanan R, Mandhukar A, Rajkumar R. Programas eficaces de cribado del cancer cervicouterino en los países en desarrollo de ingresos bajos y medios. Bull World Health Organ 2001;79(10):954-62.

2. Franco E, Duarte-Franco E, Ferenczy A. Cervical cancer: epidemiology, prevention and the role of human papillomavirus infection. CMAJ 2001; 164 (7):1017-25

3. Organización Panamericana de la Salud. Planificación de programas apropiados para la prevención del cáncer cervicouterino. Washington DC: Organización Panamericana de la Salud; 2002.

4. Robles S, White F, Peruga A. Tendencias de la mortalidad por cáncer de cuello de útero en las Américas. Bol Oficina Sanit Panam 1996;121(6):478-90.

5. Instituto Nacional de Cancerología. Registro Institucional de Cáncer. Bogotá DC: Instituto Nacional de Cancerología; 1999.

6. Muñoz N, Franceschi S, Bosetti C, Moreno V, Herrero R, Smith J, et al. Role of parity and human papillomavirus in cervical cancer: the IARC multicentric case-control study. Lancet 2002;359:1093-01.

7. Smith J, Green J, Berrington A, Appleby P, Peto J, Plummer M, et al. Cervical cancer and use of hormonal contraceptives: a systematic review. Lancet 2003; 361: 1159-67.

8. Corral F, Cueva P, Yépez J, Montés E. La baja escolaridad como factor de riesgo en el cáncer de cuello uterino. Boletín Oficina Sanitaria Panamericana. 1996;121(6):511-7.

9. Brownson R, Reif J, Alavanja M, Bal D. Cancer. In: Brownson R, Reminton P, Davis J, editors. Chronic disease epidemiology and control. Second edition. Washington DC: APHA;1998. p 335-373.

10. Ho G, Bierman R, Beardsley L, Chang C, Burk R. Natural history of cervicovaginal papillomavirus infection in young women. N Engl J Medicine 1998;338(7):423-8.

11. Ministerio de Salud. Centro Nacional de Consultoría. III Estudio de Salud bucal - ENSAB III; II Estudio Nacional de factores de riesgo de enfermedades crónicas - ENFREC II. Tomo IV: Conocimientos, actitudes y prácticas en detección temprana de cancer cervico uterino y de mama. Bogotá: Ministerio de Salud; 1999.

12. Ministerio de Salud de Colombia. Guía para la detección temprana de cáncer de cuello uterino. Resolución 412 de 2000.

13. Bosch F, Muñoz N, Sanjose de S, Navarro C, Moreo P, Ascune L, et al. Human papillomavirus and cervical intraepithelial neoplasia grade III/carcinoma in situ: a case-control study in Spain and Colombia. Cancer Epidemiol Biomarkers Prey 1993;2:415-22.

14. Sanjosé de S, Muñoz N, Bosch F, Reinman K, Pedersen N, Orfila J, Ascune L, et al. Sexually transmitted agents and cervical neoplasia in Colombia and Spain. Int. J. Cancer 1994;56:35863.

15. Selvin E, Brett K. Breast and cervical cancer screening: sociodemographic predictors among white, black, and Hispanic women. Am J Public Health 2003;93:618-23

16. Organización Panamericana de la Salud. CARMEN. Iniciativa para la prevención integrada de las enfermedades no transmisibles en las Américas. [citado 1 oct. 2003]. Disponible en: www.paho. org/spanish/ad/dpc/nc/carmen-info.htm.

17. Ministerio de Salud de la República de Costa Rica. Protocolo Proyecto CARMEN. San José: Ministerio de Salud de la República de Costa Rica;1999.

18. Departamento Administrativo Nacional de Estadística, DANE. Proyecciones municipales de población. [citado 16 enero 2004]. Disponible en: www.dane.gov.co/inf_est/series_proyecciones.htm.

19. Secretaria de Salud de Bogotá. Dirección de Salud Pública. Diagnósticos Locales con Participación Social. Localidad de Santa Fe. Bogotá DC: Secretaria Distrital de Salud de Bogotá; 1998.

20. Secretaria de Salud de Bogotá. Dirección de Salud Pública. Diagnósticos Locales con Participación Social. Localidad de Tunjuelito. Bogotá DC: Secretaria Distrital de Salud de Bogotá; 1998.

22. Hosmer D, Lemeshow S. Applied Logistic Regression. 2.a ed. New York: John Wiley \& Sons;2000.

Rev Esp Salud Pública 2004, Vol. 78, N. ${ }^{\circ} 3$ 
23. Kleinbaun D, Kupper L, Muller K. Applied regression analysis and others multivariable methods. 2.a ed. Boston: PWS-KENT; 1987.

24. Greenland S. Modeling and variable selection in epidemiologic analysis. Am J Public Health 1989;79(3):340-9.

25. Statacorp, Stata Statistical Software: Release 8.0. 2003, Stata Corporation: College Station, TX.

26. Ojeda G, Ordóñez M, Ochoa L. Asociación Probienestar de la Familia Colombiana-PROFAMILIA. Encuesta Nacional de Demografía y Salud de Profamilia. Bogotá DC: Profamilia; 2000.

26. Instituto Nacional de Cancerología. Pase la vida. Guía para el diseño de una estrategia local de comunicación del programa de detección precoz y control del cáncer de cuello uterino. Bogotá: Instituto Nacional de Cancerología;1994.

27. Newell S, Girgis A, Sanson-Fisher R, Savolainen $\mathrm{N}$. The accuracy of self-reported health behaviors and risk factors relating to cancer and cardiovascular disease in the general population. A critical review. Am J Prev Med 1999;17(3):211-229.

28. Centers for Disease Control and Prevention. Behavioral Risk Factor Surveillance System Spanish Questionnaire 1998. [citado 16 enero 2004]. Disponible en: www.cdc.gov/brfss/questionnaires/pdfques/q98cspan.pdf.

29. Corral F, Cueva P, Yépez J, Montés E. La baja escolaridad como factor de riesgo en el cáncer de cuello uterino. Bol Oficina Sanit Panam. 1996;121 (6):511-7.
30. Baker D, Middleton E. Cervical screening and health inequality in England in the 1990's. J Epidemiol Community Health 2003:57:417-23.

31. Hernández-Avila M, Lazcano-Ponce E, Alonso P, Romieu I. Evaluation of the cancer programme screening in Mexico: a population-based case-control study. Int J Epidemiol 1998;27:370-6.

32. Organización Panamericana de la Salud. Enfermedades no transmisibles. $28^{a}$ Reunión del Subcomité de planificación y programación del comité ejecutivo del consejo directivo. Washington DC: Organización Panamericana de la Salud; 1997.

33. Coughlin S, Uhler R, Hall I, PhD, Briss P. Nonadherence to breast and cervical cancer screening: what are the linkages to chronic disease risk? Preventing Chronic Disease [serial online] 2004 Jan [citado 16 enero 2004]. Disponible en www.cdc.gov/pcd/issues/2004/jan/coughlin.htm.

34. Loaiza A, Gómez S, Lucumí D. Factores sociocognitivos asociados a la práctica de la citología vaginal en mujeres de nivel socioeconómico medio y bajo de Bogotá D.C. Estudio exploratorio. Informe Final. Bogotá DC: Secretaria de Salud de Bogotá; 2003.

35. República de Colombia. Consejo Nacional de Seguridad Social en Salud. Acuerdo 008 de 1994

36. República de Colombia. Consejo Nacional de Seguridad Social en Salud. Acuerdo 229 de 2002.

37. Quinn M, Babb P, Jones J, Allen E. Effect of screening on incidence of and mortality from cancer of cervix in England: evaluation based routinely collect statistic. BMJ 1999;318:904-98. 